\title{
EL DESARROLLO MATEMÁTICO DE LA TECNOLOGÍA
}

\author{
Juan Manuel Rojas Quiñones \\ Director Programa de Formación en Matemáticas. INPAHU \\ jmrojas@inpahu.edu.co
}

\begin{abstract}
The development of human civilization has undoubtfully linked to that of handling processes and racional modification of the nature and deyices have meant a development in technology for the mathematizing of processes through which they are designed and made come true to those, wich has generated a set of intersubjectivities (interdependences and interinfluences) between mathematics and technology as strong and definite, that its genesis, development and holistic are woerth a careful study.
\end{abstract}

\section{RESUMEN}

El desarrollo de la civilización humana ha estado ineludiblemente ligado al de los procesos de manejo y modificación racional de la naturaleza, y los constructos (máquinas, herramientas) fabricados por el hombre para operativizar los mismos.

A su vez la Técnica elemental de fabricación y utilización de tales métodos y tecnofactos ha devenido en Tecnología a partir de la matematización de los procesos por medio de los cuales se diseñen y se les da realidad física a aquéllos, lo cual ha generado un conjunto de intersubjetividades (interdependencias e interinfluencias) entre la Matemática y la Tecnología tan fuerte y definitivo, que su génesis, desarrollo y holística bien valen la pena un cuidadoso estudio.

\section{INTRODUCCIÓN}

El presente trabajo nació de un cuestionamiento de orden pedagógico acerca de la pertinencia de la Matemática en la educación formal, que se puede especificar en la pregunta: ¿por qué es importante la Matemática en un currículo tecnológico?

Mi área es la Matemática, y además manejo con alguna propiedad la Pedagogía — dos de los tres elementos que componen el sustrato de la pregunta- pero en el campo tecnológico mis conocimientos para entonces dejaban mucho qué desear. Obviamente, hubiera podido esgrimir una respuesta versátil (con todo lo de ambiguo que tienen las respuestas versátiles): "No se concibe absolutamente ningún currículo educativo sin un basamento, tácito o explícito, de orden matemático", pero a nadie que maneje medianamente el tema le hubiera satisfecho semejante respuesta.

Muchos meses después de haber sido formulado el anterior cuestionamiento aún no he llegado a convertirme en un experto en tecnología, pero, a fuerza de cavilación, consulta y 
discusión pertinentes, creo haber logrado una aproximación bastante aceptable al tipo de relación que histórica y epistemológicamente ha vinculado la Matemática con la Tecnología, y con ello espero haber logrado un ensayo suficientemente concreto y coherente como para que cualquier persona interesada que lo siga, obtenga de él elementos de fundamentación lo bastante sólidos como para que logre, a partir de ellos, - o si lo prefiere en contra de ellos, que esa es otra manera de utilizar bien los interrogantes- elaborar su propia respuesta.

Si no hablo de proporcionar yo mismo tal respuesta, es simplemente porque, en primer, lugar no creo en las respuestas de cubrimiento ecuménico y, en segundo término, porque estoy convencido de que más aporta a la formación intelectual de una persona un planteamiento polemizable, una duda bien estructurada, y una pregunta adecuadamente formulada.

Siendo la relación Matemática-Tecnología tan antigua como lo es la capacidad de raciocinio del hombre, y teniendo esta relación el peso de la responsabilidad de, a su manera, describir éste, su estudio minucioso no es concebible en un espacio tan reducido como lo es por su misma naturaleza un artículo de revista, sino que en su tratamiento requiere mayor extensión si no se quiere sacrificar su profundidad.

Es así como el presente artículo sólo pretende avanzar en el análisis de esta interacción heurística hasta el punto que el complejo y acelerado estadio de la civilización de finales del siglo XIX le exige al hombre racionalizar y agilizar los procesos informáticos.

El desarrollo de la Comunicación y el posterior de la Telemática, quedan de momento por fuera de los alcances de este escrito.

\section{LA PREHISTORIA}

El primer contacto del hombre con la Matemática tuvo que haber sido la observación de la luna llena, a la cual accedió cuando se hizo homus erectus y pudo levantar la mirada del suelo.

Si algunos animales superiores, tales como los cánidos, manifiestan clara atracción y hasta reverencia hacia la luna llena, mayor hubo de ser el asombro del hombre primitivo por esa primera -y única durante milenios- expresión de simetría perfecta. (No cuentan para estos propósitos los casos de simetría bilateral de casi todos los seres vivos y hasta del arco iris, por carecer del mismo nivel de perfección que ostenta el círculo).

El círculo tiene entonces la opción de ser la primera apreciación matemática del hombre, y no es de extrañar que la concretara en la redondez de las vasijas de arcilla que comenzaba a fabricar, facilitada ciertamente por el movimiento y posición natural las manos en su elaboración. No es que el círculo le fuera realmente ajeno antes (se sentaban los hombres formando una circunferencia para conversar) sino que observarse a si mismo es mucho más complicado que observar los fenómenos exteriores a uno.

La primera manifestación de inteligencia superior del hombre -en cuanto requiere una alta dosis de abstracción- la constituye la elaboración de dibujos en las paredes de las cavernas que habitaba en sus primeras épocas, y no sólo es admirable el dibujo en sí mismo sino aún más su intencionalidad: el hombre propone mediante sus pinturas rupestres que lo representado queda endosado; la representación constituye un aval de 
dominio sobre lo representado. Este principio, que es ni más ni menos la materialización gráfica del juego del poder que regula y, de cierta manera, explica la existencia biológica y las relaciones ecológicas que se dan en su interior, lo acompañará ya por siempre y es la fundamentación epistemológica de los planos, diseños, maquetas, prototipos y esquemas que irá desarrollando a través de la historia y que hoy día hace pensar al hombre que lo desarmado en sus partes componentes es construible y por tanto poseíble.

Claro, para llegar a la matemetización formal de este hecho, el hombre tuvo que esperar milenios, y pasar primero al menos por los estudios de proporcionalidad y semejanza de Thales, por el método de Arquímedes, por la Geometría Proyectiva de Descartes - urgida por los pintores del Renacimiento para suplir necesidades de su oficio- y por la Geometría Analítica de Descartes en la misma época (Koyre A. 1981) pero ya la representación gráfica de un objeto (coincidente o no con él) es un firme comienzo de homotecia, esto es, de matematización de la realidad, de apropiación de la realidad, pues es indudable que el hombre matematiza el mundo para apoderarse de él.

Este es el segundo hito en el desarrollo del pensamiento humano, si aceptamos como primero la "herramentización" de sus manos que hace el hombre, el manejo más o menos independiente de sus dedos, la conciencia de que pueden usarse como herramientas, para fabricar herramientas y para hacer uso de ellas. En este orden de ideas, cabe decir que esa es la intencionalidad, consciente o no, de la nominación de las cosas y por ahí mismo de su definición: lo nombrado (representado verbalmente, en principio, y escrituralmente después) es poseído. Se define un fenómeno para ser dueño de él, para dominarlo.

Y ya que estamos hablando de definiciones y de Matemática, nada más oportuno que intentar una definición de la misma. Acerca de las definiciones hay toda una teoría filosófica, pero lo esencial para nuestros propósitos es lo siguiente: si yo quiero poseer y dominar un conocimiento, debo tener una definición suya, y entre más auténtica sea esa definición, más me apropiaré de dicho conocimiento. En concreto, yo defino la Matemática como la disciplina mental dedicada al estudio metódico y detallado de la Relación. Esta Relación se da entre los entes que conforman la realidad perceptible y/o imaginable. Estando ya en esto, me parece pertinente definir también Tecnología: técnica discursiva, es decir, técnica matematizada intencionalmente.

El tercer hito en el desarrollo intelectual humano lo constituye la invención del lenguaje articulado. Se dice, un poco socarronamente, que la mayor habilidad oral de la mujer se debe a que, mientras el varón primitivo en sus faenas de cacería y acechando su presa, debía guardar el más absoluto silencio y desarrollar más bien su capacidad de raciocinio, de anticipación mental a los movimientos y trayectorias del animal perseguido, durante horas y hasta días, la mujer, quien se ha quedado en la aldea a cargo de las labores domésticas, cuenta con más libertad y puede dedicarse a perfeccionar su recién adquirida propiedad parlante mientras desarrolla labores eminentemente manuales, es decir, mecánicas y que requieren poca concentración mental. Los antropólogos dirán qué de cierto tiene esta versión de la historia del desarrollo diversificado de la racionalidad de los seres humanos según un criterio de diferenciación de géneros sexuales, pero se ve bastante apropiada.

A partir de estas apreciaciones no es desencaminado afirmar que esa es la naturaleza de la desigualdad intelectual del hombre y la mujer: la disparidad de origen de sus respectivas racionalidades: la femenina de tipo oral (inductiva) y la masculina de tipo reflexivo (deductiva). Hoy día es imposible categorizar dichas racionalidades; la pregunta 
de cuál de los dos es superior no admite respuestas ya que no se pueden comparar en igualdad de condiciones, según los mismos parámetros.

De una u otra manera, es la mujer quien tiene la oportunidad de hacer el primer gran descubrimiento de la humanidad: las plantas se reproducen por sí mismas y lo hacen a partir de constituyentes de ellas mismas. Entonces, no hay que andar detrás de "nuevos pastos" cuando se han agotado los disponibles en el lugar temporalmente habitado, sino reproducirlos ahí mismo, in situ. Esto les origina sentido de propiedad, de pertenencia, les permite construir viviendas más sólidas que resistan no sólo los ataques de sus enemigos sino también los embates del tiempo. Ese sentido de propiedad, por necesidad, es más fuerte en la mujer que en el hombre. La mujer inventa el sedentarismo pero le queda muy difícil obligar al hombre a que lo acepte para él. Aunque casi paralela a la agricultura nace la ganadería, haciendo obsoleto el oficio de cazador del hombre, éste no cree que sea actividad varonil quedarse en cosa todo el día dedicado a labores domésticas y entonces inventa la guerra, cacería de productos terminados, en la cual gana o pierde sus pertenencias sin que esto último represente para él una tragedia, pues con la guerra le nace el espíritus de jugador, de explorador; para la mujer silo es. De todas maneras no todos los hombres son aptos para la guerra y algunos se quedan en cosa colaborando con las labores agrícolas, pero no pueden echar en saco roto las habilidades mentales adquiridas con tanto esfuerzo durante tantos milenios, y las pone al servicio de la agricultura racionalizándola. Es de suponer que así, por un principio de economía, nace la idea tanto de rectángulo como de paralelismo: una sementera es más manejable y productiva si se compone de hileras de plantas paralelas y de igual longitud. También es deseable que las plantas de una misma hilera estén situadas a igual distancia unas de otras, y entonces los rudimentos de la recta numérica, y de su representante material, la regla, comienzan a perfilarse dando lugar, indudablemente, a los inicios del conteo. Este conteo debió haber sido trasladado, como primitiva contabilidad, al control de inventarios de semovientes y otras pertenencias (Baldor A., 1996).

Estas actividades, definitivamente manuales y de categoría técnica, dan origen a los sistemas de numeración. Inicialmente los sistemas de numeración tienen una génesis antropométrica. Es curioso observar que, mientras el hombre euro-asiático, quien ya ha cubierto sus pies con calzado rudimentario para combatir el frío, desarrolla un sistema decimal de numeración, los mayas, quienes en un clima más benigno andan preferentemente descalzos (sus "guaraches" o sandalias son usados sólo por los altos dignatarios y de todos modos dejan al descubierto los dedos de los pies) utilizan un sistema vigesimal. El sistema sexagesimal de los babilonios -que lo combinaban con el decimal—, tiene explicaciones más de orden mágico (Aaboe A.,1964).

El hombre, entonces, sin proponérselo y sin siquiera tomar conciencia de ello, inventa los inicios de o que será mucho tiempo después la Teoría de Conjuntos y con ella el concepto de número cuando pone en relación uno a uno los elementos más múltiples y manejables de su propia humanidad, los dedos, con los elementos externos sobre los cuales desea ejercer control y poder: plantas, reses, vestiduras, utensilios, etc., convirtiendo de paso sus manos de herramientas meramente mecánicas en herramientas intelectuales. Así, de una manera y con un origen netamente técnico, hace su aparición la Aritmética y unos primeros rudimentos de Geometría (Aaboe A., 1964).

Como ya se mencionó, el desarrollo de la agricultura tuvo que haber dado origen también a la primera herramienta matemática material: la regla. No es difícil suponer a los agricultores primitivos delimitando sembradíos por medio de cuerdas tirantes (o varas largas en su defecto) pobladas de nudos simétricamente distribuidos con un significado 
matemático implícito aunque seguramente no claro para ellos: el concepto de unidad de longitud, y con él el concepto de metrización del mundo físico (Gallego R.,1995).

Las viviendas que conformaban los poblados debían estar ubicadas en forma de circunferencia para que todas dispusieran de la misma visibilidad hacia el exterior de la aldea, y además esta fuera exhaustiva, con miras a poder vigilarla más eficientemente. Por otra parte, haciendo una lectura intuitiva de un principio de economía natural, los hombres en la noche, esto ya se mencioné, al sentarse alrededor del fuego y no sólo para calentarse sino sobretodo para intercambiar experiencias, conocimientos y planear acciones sociales a seguir, se sientan formando una circunferencia con su centro perfectamente definido: la hoguera. Pero es una circunferencia situada en un plano horizontal y a nivel de suelo, sin muchas posibilidades cinemáticas; podría haber sido una circunferencia perfectamente inmovible a través de milenios si no fuera porque alguna vez incité al hombre a la danza, dándose a si misma rotabilidad, primera transformación geométrica, pero colocar esa circunferencia en forma vertical es una transformación geométrica que, si bien es otro tipo de rotación, no es sólo un imposible físico en el momento sino que requiere un poder de abstracción tal que el desarrollo intelectual de entonces aún no lo permite. Necesariamente el arco de circunferencia sí es no sólo conocido sino ampliamente manejado por el hombre: ha visto el arco iris, ha construido arcos para lanzar flechas, cuando ha intentado trazar una recta sobre una roca vertical o una pared aquella se le ha ido inclinando hacia abajo si ha dejado los pies en el mismo sitio, etc., pero la noción de circunferencia, y principalmente sus propiedades cinemáticas, todavía no le son presentes (Hull L., 1981).

Pero los ríos redondean las piedras, cierta clase de ellas, las convierten en "cantos rodados" y el hombre, por necesidad habitante de sus orillas, por necesidad tiene que observar este fenómeno. El rodamiento de objetos no será nuevo para el a estas alturas si tenemos en cuenta que tuvo que haber observado muchas veces el despeñamiento de animales ( $\mathrm{y}$, por qué no, de congéneres) en las laderas más pronunciadas de las montañas, los deslizamientos de piedras y nieve en las mismas, pero estos rodamientos no estaban asociados con el transporte ya que cada objeto rodaba por sí mismo, no portando a otro. Es mi parecer que el rodamiento, o mejor la capacidad de rodamiento de los cantos rodados, tiene connotaciones distintas: si uno pisa por descuido un canto rodado, éste lo transporta por algunos centímetros. Claro, al final caerá estrepitosamente con tanto disgusto y dolor que muy posiblemente no quede en condiciones de filosofar sobre el fenómeno; pero si no es uno quien lo sufre sino quien lo presencia, el asunto tiene más posibilidades epistemológicas: con un poco que se ponga a pensar descubre el principio de rodamiento e inventa la rueda. No una rueda cilíndrica, como la que tuvo que haber colocado en los primeros carromatos (¿troncos?) (Hull L.,1981) sino una rueda más bien esferoidal y totalmente desprovista de eje, que éste sí es un invento muy del hombre, que tuvo que ingeniar para hacer realmente útil su descubrimiento.

El invento del eje le tiene que traer necesariamente la idea de simetría central, la observación de que, en su permanente caída - que no otra cosa es rodar- todos los puntos del círculo que conforman la rueda se mueven repetitivamente, menos el punto central que es donde está ubicado el eje. Ya nunca más podrá el hombre disociar la circunferencia de su centro, así en términos de continuidad le sea extraño.

Pero el invento de la rueda tiene más implicaciones que las meras técnicas y matemáticas. Pienso yo que da origen a la Filosofía al proporcionarle al hombre la primera noción de infinito (Wittgeinstein L., 1994); su repetitividad tuvo que haber provocado en la mente humana la idea de ciclismo de los fenómenos naturales (de hecho está la doctrina 
del Eterno Retorno de Nietzche) y es por todo esto por lo que a la circunferencia, tanto como su contenido, el círculo, se le atribuyeron desde siempre propiedades mágicas. Para entonces el hombre tiene ya bastante geometrizado el mundo y está listo para dar a luz civilizaciones como la egipcia y la sumeria.

\section{LA ANTIGÜEDAD}

Es la propiedad circunferencial de la rueda, esa eterna caída, la que facilita el transporte de objetos, en particular de pesados materiales para construir ciudades. La arquitectura babilónica es bastante rectangular, lo cual tuvo que haber impulsado el desarrollo de instrumentos de trazo corrientes: la regla dotada de unidades de longitud aceptadas mediante algún tipo de acuerdo social y de todas maneras antropométricas (geme, cuarta, codo, brazada, pie, etc.) (Baldor A., 1996) que no pocos problemas tuvieron que haber suscitado al querer trasladarlos de un lugar a otro, sobretodo si estos lugares eran habitados por etnias bien diferentes; la escuadra, la plomada, el compás en su doble uso: trazar circunferencias y trasladar medidas de longitud- etc.

Mas la construcción, al igual que la agricultura pero con mayores exigencias de exactitud en la determinación de extensión y forma, no utiliza sólo longitudes sino también áreas, y aparece entonces, como una necesidad eminentemente técnica, la multiplicación (la suma y la resta, operaciones que en honor a la verdad no requieren grandes esfuerzos mentales, ya habían aparecido mucho antes como satisfactores a necesidades de orden comercial). La multiplicación es en realidad una operación mucho más compleja de lo que parece; eso de que es una suma abreviada de sumandos iguales, tiene tanto de cierto como de inexacto. Trabajando con elementos de la realidad inmediata como manzanas (cinco grupos de tres manzanas cada uno nos da un total de quince manzanas, luego podemos concluir que $3 \times 5=15$, pero dejando por fuera la determinación de los elementos sobre los cuales se opera -manzanas- pues multiplicar manzanas por manzanas carece de sentido; podemos multiplicar cardinalidades pero no objetos en sí mismo ¡manzanas por manzanas nos daría manzanas cuadradas!) esta aseveración resulta aceptable, pero en cuanto pasamos al conjunto de las distancias -y ni hablar de los vectores - las cosas comienzan a ser bastante menos claras, pues el producto, verbi gracia, de la multiplicación de $5 \mathrm{~cm}$ por $7 \mathrm{~cm}$ no es simplemente $35 \mathrm{~cm}$, sino $35 \mathrm{~cm}^{2}$, ¡multiplicando longitudes conseguimos áreas, que en rigor están mucho más emparentadas con los vectores que con los escalares!... y así podemos continuar hasta conseguir dimensiones insospechadas. Esto para nosotros hoy día es muy elemental; usualmente ni siquiera nos detenemos a pensar en ello cada vez que multiplicamos, pero para los primeros constructores y agrimensores profesionales, tuvo que haber significado un gran esfuerzo mental por ser este un hecho que se sale del desarrollo natural de la matemática. Sin embargo de alguna manera lo superaron y con la multiplicación sentaron las bases del desarrollo de las ecuaciones no lineales, en cuyo estudio avanzaron bastante los babilonios (Aaboe A., 1964).

Se dice que los egipcios desconocían el teorema de Pitágoras (Hull L., 1981). Parece poco probable esta afirmación si tenemos en cuenta que sus famosas construcciones en forma piramidal exigen un cuidadoso manejo del triángulo rectángulo (es mi parecer que los egipcios escogieron la pirámide como estructura formal de sus construcciones religiosas porque aquella representa la forma, vista desde abajo, que tendría una construcción paralelepípeda elevada hasta el sol, esto es, la pirámide es el simulacro físico de la ambición del hombre de llegar a Dios). En cualquier caso las construcciones 
se hacen sobre planos y maquetas, si bien tal vez aún no metrizados, pero ya era visible en ellos la idea de homotecia premeditada, del principio de escala.

Y así llegamos a Grecia. Thales de Mileto era un inquieto viajero que tuvo ocasión de acceder a todos esos conocimientos matemáticos manejados por los egipcios y babilonios y los llevó a su patria. Ciertamente no se contenté con poseer y difundir dichos conocimientos sino que se propuso desarrollarlos menos pragmáticamente, según el pensamiento griego, y funda así la Matemática como una ciencia pura, pero lo que de ella le interesa realmente es la Geometría, y, dejando de lado tanto su aplicaciones como los interesantes desarrollos algebraicos alcanzados por los babilonios. Thales sienta las bases de la Geometría Proyectiva con su famoso teorema de la proporcionalidad, pero como no era una necesidad sentida por la sociedad de entonces, no es entendida de esta manera (Hull L., 1981). Por lo demás, a la Matemática griega no le aporta nada la técnica, si tenemos en cuenta que ésta es considerada de bajo nivel intelectual o propia sólo de gentes del pueblo, que eran precisamente quienes no trabajaban la Matemática, ciencia filosófica y espiritual por excelencia. Las cosas se complican con la aparición en escena de Pitágoras, una de las más insignes figuras de la Matemática de todos los tiempos... y a quien, en honor de la verdad, muy poco le debe la Matemática si consideramos que el famoso "teorema de Pitágoras"es conocido y manejado por los babilonios mil años antes de su nacimiento, junto con los números y las tablas pitagóricas. Bueno, sería demasiado desagradecimiento no reconocer que la Matemática le debe a Pitágoras su nombre, aunque para él Matemática era todo conocimiento sistematizable para su estudio; pero más le deben la Física y la Filosofía. La Física por haber sido Pitágoras el primer físico experimental (contradiciendo, claro está, sus convicciones filosóficas). Mediante experimentos sucesivos determiné la relación entre las longitudes de las cuerdas de instrumentos musicales que producen combinaciones armónicas de sonidos. Y la Filosofía por haber instaurado el primer sistema filosófico serio de la historia, precisamente el Pitagorismo (Hull L., 1981).

\section{LA HISTORIA}

La escuela pitagórica se encuentra con una anormalidad inadmisible para el concepto de perfección del número: hay cantidades (raíces cuadradas, cúbicas, etc.) que no pueden ser expresadas como la relación (razón) de dos números enteros. Esto no es razonable, es sencillamente irracional. Tales aberraciones deben ser expulsadas de la Matemática, aún a riesgo de perder con ellas desarrollos imprescindibles de esta ciencia, en particular el Algebra. Y lo hacen evadiendo la cuantificación, geometrizando a ultranza la Matemática (Hull L., 1981), así hechos tan patentes como la relación entre radio y circunferencia, entre catetos e hipotenusa estén poniendo de presente, casi gritando que esa era una salida en falso, Pero así sobrevive la Matemática durante cuatro siglos, hasta que aparece Arquímedes, el más grande matemático de todos los tiempos, re-fundador de la Matemática y fundador de la Tecnología.

Arquímedes le pierde el miedo a los "números irracionales", le pierde el respeto a la cerrada Filosofía griega y comienza a aplicar los desarrollos matemáticos a la construcción de entes mecánicos (Gamov G., 1971). La metrización desde entonces es una dimensión válida de la Matemática, y a partir de ella ésta se puede "rebajar"al mundo físico, puede mezclarse con la materia sin degradar-se, más bien enriqueciéndose. Geometrizando la materia, y no sólo la idea de materia, Arquímedes construye bombas de agua, polipastos, palancas, catapultas, arietes, lentes... cuanto su febril imaginación y las limitaciones técnicas y materiales del momento le permiten (Gamov G., 1971). 
Desafortunadamente sus más importantes escritos, entre ellos el "Método", se pierden para la humanidad durante casi 23 siglos; por eso los pensadores del Renacimiento tienen que "ponerse a descubrir el agua tibia".

A Grecia le sucede Roma, pero Roma no tiene necesidad de pensadores, ya que los que necesite para su pujante desarrollo comercial, urbanístico y marcial los puede traer de Grecia, donde la Filosofía se da silvestre. La Matemática prácticamente fallece por inanición. Durante más de un milenio la humanidad se comercializa, esto es, sólo exige desarrollos de orden económico. Se dice que el único aporte de los romanos a la Matemática es la invención de la unidad de longitud llamada "pulgada" (Baldor A., 1996); no es fácil creer que haya sido tan pobre su desempeño intelectual, pero así lo aseguran los historiadores. Claro, tiene que haber algunos desarrollos en agrimensura, en contabilidad, pero la historia no nos muestra que haya habido más.

\subsection{El Renacimiento}

La Matemática, huérfana de pensadores y practicantes, toma entonces nuevos rumbos. Comienza a florecer en la India, pasa a Arabia y regresa a occidente después de haberse fortalecido el Algebra, de haber sido inventado el cero y de haberse racionalizado grandemente su natación numérica.

El Renacimiento merece realmente su nombre, pero no porque, como lo creían muchos para entonces, la civilización haya retornado a nacer sino porque ha nacido OTRA vez. Durante toda la Edad Media la técnica ha sido una práctica eminentemente artesanal, sin grandes pretensiones; se cultiva "para ganar el pan de cada día". Pero la ciencia despierta y se encuentra con la Matemática recién importada de oriente y exige nuevos desarrollos mecánicos en qué apoyar sus postulaciones: aparatos experimentales, instrumentos de medición, y entonces la Técnica deviene nuevamente, y ya para siempre, en Tecnología, como socia ineludible del desarrollo científico, del desarrollo industrial, del desarrollo intelectual de la humanidad (Koyre A., 1981).

\subsection{El caso de la igualdad}

Indudablemente dentro de las relaciones que se dan entre entidades matemáticas, es la igualdad la más importante. No se ha podido averiguar en qué momento el hombre establece la relación de igualdad, que es de suponer tuvo que haberse iniciado con un principio de similitud entre objetos de la realidad cotidiana tales como frutos, animales, hojas de plantas, vasijas etc., principio claro de clasificación en clases; luego llegaría la identidad como caso extremo de similitud (gemelos, verbi gracia) sin llegar aún, claro está, a la propiedad reflexiva, que partirá de la invención del espejo y recorrerá un camino muy tortuoso en la mente del hombre para que puede éste concluir que es igual a sí mismo. La igualdad, como propiedad exclusiva de los números, es evidentemente un concepto filosófico que sólo los griegos estarán en capacidad de enunciar; hacer trascender estas observaciones y sus respectivas conceptualizaciones a la idea de número, requiere milenios y un avance muy grande del pensamiento humano. De todas maneras, y a juzgar por el concepto de "ecuación", presente ya en la Matemática babilonia, la idea de igualdad ya estaba en la mente del hombre unos 20 siglos antes de Cristo. Para entonces los babilonios manejaban ecuaciones, y ya éstas tenían la connotación de igualdad de cantidades, esto es, la idea de número era ya bastante clara como abstracción de cantidad (Aaboe A., 1964). 
El signo que actualmente utilizamos para expresar la relación de igualdad entre cantidades aparentemente disímiles, tuvo que esperar hasta el siglo XVI para ser inventado por Robert Recarde (Baldar A., 1996), pero ya en tiempos de los babilonios tenía que estar claro que la igualdad es una propiedad exclusivamente numérica, y que cualquier otro caso de similitud extrema puede ser llamado "congruencia" o "coincidencia" pero no "igualdad".

Estamos tan acostumbrados al uso de la igualdad para solucionar asuntos desde los más elementales de la Aritmética común hasta los más intrincados de la Mecánica Cuántica, que no nos paramos a pensar en su significado, de lo contrario la mente humana más esclarecida se quedaría perpleja cuando le presentaran por primera vez, verbi gracia, esta igualdad:

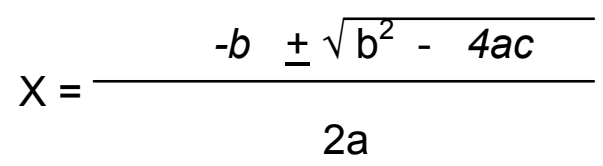

puesto que aquí la idea intuitiva de igualdad (ser como dos gotas de agua) está lejos de parecer inmediata y trivial. A lo más podríamos decir sobre ella que hay una identificación potencial, fruto futuro de un dispendioso trabajo que nos será premiado con la resolución de dicha ecuación. Sin embargo corrientemente no le metemos filosofía al asunto y más bien optamos por creerlo perezosamente cuando alguien confiable nos lo dice.

A mí me parece que hay mucho de abuso del concepto de igualdad en una fórmula matemática, lo mismo que de la credibilidad del público, pero sin duda la igualdad lo resiste bien y el público en general prefiere creer a pensar.

Partiendo de la igualdad, y si le quitamos al término "fórmula" la connotación de "receta", para darle la más obvia de "formulación de un concepto", tendremos un mecanismo multifuncional que lo mismo nos describe, que nos define, que nos ofrece un procedimiento expedito para, en primera instancia, desarrollar procesos y, como culminación, lograr canstructos terminadas. Es en el momento en que el hombre entiende esta propiedad matemática, en el cual el artesano se vuelve tecnólogo.

Es Galileo (Koyre A., 1981) quien, ignorando los desarrollos de Arquímedes, primera matematiza oficialmente los fenómenos físicos, basada en los trabajos de sus predecesores y maestros Bonamicci y Benedetti. Su capacidad explicativa y su oportunismo histórico (en el buen sentido) lo convierten en el adalid de la moderna ciencia; es él quien estatuye el procedimiento de formulación de la realidad en términos matemáticos con el fin de controlarla, esto es, se plantea un enigma -en términos de incógnita - que par sí mismo no es manejable, pero a continuación presenta un hecho enmascarado aunque igual y ciertamente manejable (¿data de ontología a un hecho reacio a ser realizable?). La $X$ de la ecuación anterior no nos permite par sí misma adentramos en sus estructuras y significados, pero su homólogo

$\frac{-b \pm \sqrt{ } b^{2}-4 a c}{2 a}$

sí, nos describe todo un procesos operativo, nos muestra los componentes de la $X$ desnuda, nos describe un mecanismo, un procedimiento teleológico que nos conduce no 
sólo a un conocimiento sobre dichas componentes de la $\mathrm{X}$, sino a la manera de armarla $\mathrm{y}$ desarmarla, y/o a partir de ella construir alga nueva (par ejemplo una gráfica) esta es, desnaturalizaría.

La que hace el hombre cuando formula matemáticamente la realidad, es demostrar que no ha perdido aún esa herencia de sus primeros antepasadas, ese creer que las cosas tienen un nombre el cual hay que descubrir porque la cosa nombrada es cosa poseída, ya que los objetos, animistamente, responden a su nombre. La formulación matemática de un objeto o hecho va, claro está, mucha más allá: propone que la cosa descompuesta en sus partes elementales es cosa realizable y por tanto poseíble.

Es Descartes quien entiende mejor todo esto, y es él quien realmente opera el tránsito del mundo ya geometrizada, pero metafísicamente, parlas griegas, al mundo operativo algebnizado de la saciedad tecnológica que surge a partir de entonces. Y es que es el Algebra -Aritmética venida a más - la rama más operativa de la Matemática, tanto por su capacidad descriptiva, analítica —si se quiere- y sintetizadora — también si se quiere - como por el uso ya descrita que hace de la igualdad. La Geometría Analítica de Descartes (Babini J., 1974) inicia la descripción y metodología de trazado de figuras ideales, trayenda al mundo material la elipse, la parábola, la hipérbola, la catenaria, el conoide, la bruja... y muchas más curvas que las idealmente tratadas por los griegos antes y después de Apolonio; y las pone al servicio de la tecnología, ya sea describiendo trayectorias (tiro parabólica, recorrido de los planetas) propiedades de la reflexión del sonido (elipse) relaciones entre circunferencia y diámetro (movimiento armónico simple), en fin, toda la mecanización de que eran capaces los activos técnicos de entonces elevados ya a la categoría de tecnólogos precisamente por el re-descubrimiento y utilización masiva de estas propiedades de la matemática.

También las ciencias administrativas se favorecen de este auge matematizador del Renacimiento, cuando Lucas Pacioli inventa la contabilidad por partida dable y Blaise Pascal (Varias, 1974), para satisfacer al caballero de Mere, jugador empedernido, inventa la Probabilidad, fundamento prospectivo de la Estadística - ciertamente ya existente pero no formalizada- que dotan a las ciencias administrativas de instrumentos confiables y expeditos para todas las procesos de registro, descripción, control y predicción de hechos económicos y administrativos.

La Geometría Proyectiva había estado latente durante toda la historia de la civilización, pero ésta no le había brindado la oportunidad de manifestarse como tal y ofrecer todo su potencial tecnológico. Es en el Renacimiento, ya con más apremios de orden tecnológico, cuando el hombre, a través de Gerard Desargues (Varios, 1974) la intenta de nuevo, esta vez con éxito, y fundamenta así con piso firme ese ancestral deseo del hombre de representar a escala la realidad presente o futuro. Planos, maquetas, diseños, prototipos, esquemas, no sólo son ahora instrumentos totalmente confiables sino de extendida uso. El hombre puede a través de ellos ver hecho realidad, con gradas de precisión inimaginable antes, la que su creatividad le dicta: basta encontrar la fórmula matemática adecuada que le describa y explique su idea. Se abusa, clara está, y entonces las fórmulas matemáticas adquieren connotación de fórmulas mágicas, pero es superior el cientificismo que el magicismo que se les otorga y se convierten, ya por siempre, en auxiliares imprescindibles del desarrollo de la civilización humana.

Paralelo a la Geometría Analítica surge el Cálculo. Simultáneamente en Alemania Leibniz (Varias, 1974), buscando satisfacer necesidades de orden economicista y hasta filosófica, inventa el cálculo diferencial; en Inglaterra, par requerimientos más de las 
ciencias naturales, Newton hace lo mismo. Es la variación del cambio en el comportamiento (más exactamente la variación de la "razón"de cambio) de curvas geométricas no circunferenciales lo que motiva el desarrollo de esta nueva rama de la Matemática. Más tarde se utilizará para determinar áreas de figuras irregulares, volúmenes de cuerpos, trabaja consumida en una labor, máximos y mínimos de áreas necesarias, —par ejemplo para imprimir la página de un libro en la también recientemente inventada imprenta-, etc. En definitiva el Cálculo pasa muy pronto a ser herramienta conceptual muy allegada a la ciencia, la ingeniería, la industria y toda aquella actividad humana susceptible de ser "calculable".

En el Renacimiento había que ser sabio integral. La eran Newton, Pascal, Descartes, Galileo, Leibniz, y lo era Huygens (Koyre A., 1981), quien no queriendo quedarse atrás del arrollador impulso de la tecnología, inventa la primera máquina -instrumento que habría de llegar a todos los hogares del mundo conocido: el reloj de péndulo, dotado de mecanismo que acumulaban y dosificaban la utilización de la energía y se sustraían a los caprichos del tiempo imperante en el lugar. No hay que olvidar que el péndulo es una de las fascinaciones del renacimiento y una de las más importantes motivaciones científicas de Galilea.

De la Geometría Prayectiva deviene, mediatizado por el Cálculo, otro gran invento de la época y otro importantísimo auxiliar de la tecnología, la Geometría Diferencial. El asunto no para ahí, por supuesto, que o si no el hombre dejaría de ser hombre, pues muy pronto éste descubre que los objetos percibidos a través de una proyección, fundamento de la Geometría Prayectiva, se deforman al cambiar de posición el plano en el cual se proyectan, originando nuevos objetos y nuevas pasibilidades tecnológicas: la circunferencia puede ser convertida en elipse, el rectángulo en trapecio, etc. $\mathrm{Y}$ de aquí nace una nueva rama de la Geometría llamada Topología (Varias, 1974), que no es otra cosa que la Geometría de la deformación (sin incluir rupturas) que amplía inconmensurablemente las pasibilidades tecnológicas al crear tanto nuevas formas como nuevos mecanismo para crearlas, descubrirlas y construirlas. Luego aparecerá la Topología diferencial... y es muy difícil saber hasta dónde se puede avanzar por este camino.

Pero hay otro camino importante qué recorrer. La idea de utilizar medios mecánicas o semiautomáticos para realizar cálculos aritméticos es muy antigua. El origen del ábaco se pierde en la antigüedad, y computadores de alguna clase fueron efectivamente construidos por los antiguos griegos. Blaise Pascal (Varios, 1974), en el siglo XVII, construyó un mecanismo que funcionaba para realizar operaciones aritméticas. En ese mismo siglo John Napier (Varios, 1974) (inventar de los logaritmos) tuvo menos suerte, pero también lo intentó. Gottfried W. Leibniz (Varios, 1974) uno de los creadores de la Lógica Matemática y co-inventor del Cálculo, desarrolló un programa para lo que debería ser llamado "el pensar automatizado". Además creó el sistema binario de numeración, base de cualquier lenguaje de computación hay día. Pero el hombre que vio más claramente la realización de un computador general, completo y provisto de un esquema de programación flexible y de unidades de memoria, fue Charles Babbage (Varios, 1974) de Inglaterra, quien en 1833 descubrió una máquina que él llamó "la máquina analítica". Pasó el resto de su vida y gastó gran parte de su fortuna tratando de construirla sin conseguir un éxito completo, la que hacia el final de sus días le hizo expresar:

"no he tenido un solo día feliz en toda mi vida". 


\section{A MODO DE CONCLUSION}

Una de las maneras como se define la Matemática es diciendo que ella es el lenguaje en que esta escrito en universo y, en ese orden de ideas, el conjunto de algoritmos que la describe, explica y lo hace funcionar para el hombre.

Más allá del fondo teológico de esta definición está la dificultad, perfectamente determinada por Karl Popper (1996) de establecer lenguajes sintéticos (impersonales y universales) que permitan, precisamente, describir objetivamente la fenomenología del universo y por esa vía descomponerlo en sus partes constituyentes, entenderlo y manejarlo.

En cualquier cosa, aun no siendo la Matemática ese lenguaje utópico, es innegable que sí posee relevantes cualidades hermenéuticas y heurísticas que durante toda la historia de la humanidad han fungido como trasfondo e hilo conductor del desarrollo de ésta.

\section{BIBLIOGRAFIA}

Aaboe Asger: "Episodios históricos desde Babilonia hasta Ptolomeo". Cali: Editorial Norma, 1964.

Arquímedes: “El método”. Buenos Aires: Eudeba, 1966.

Babini, José. "Historia de las ideas modernas en matemática", Organización de los Estados Americanos, Buenos Aires, 1974.

Baldor, Aurelio. "Aritmética", sin pie de imprenta.

Gallego Badillo, Rómulo: "Discurso constructivista sobre las tecnologías". Bogotá: Editorial Libros y Libres, 1995.

Gamov, George. "Biografía de la física”. Navarra: Biblioteca general Salvat, 1971.

García Bacca, Juan David. "Elogio de la técnica". Barcelona: Editorial Antropos, Barcelona, 1987.

Hull L.W.H. "Historia y filosofía de la ciencia". Barcelona: Ariel, 1981.

Koyre, Alexandre. "Estudias galileanos”. México: Siglo XXI Editores, 1981.

Popper, Karl. "La lógica de la investigación científica”. México: REI, 1996.

Varios. "Matemáticas en el mundo moderno". Madrid: Editarial Blume, 1974.

Wittgeinstein, Ludwing: "Tractatus Logico Philosophicus". Barcelona: Editorial Altaya, 1994. 